\title{
THE NATURE OF SHOCKS IN MOLECULAR CLOUDS
}

\author{
Peter W. J. L. Brand \\ Department of Astronomy,University of Edinburgh \\ Royal Observatory Edinburgh EH9 3HJ, U.K.
}

\begin{abstract}
Evidence is presented to suggest that the shocked molecular hydrogen emission in the brightest part of the Orion outflow is produced in a J-shock and not a C-shock; that this is true throughout the entire flow; that it may be true in many outflow sources; and that this exacerbates problems with current explanations of the very wide velocity profiles observed in molecular hydrogen emission.
\end{abstract}

\section{THE DATA}

This paper summarizes work done at the U.K. Infrared Telescope (supported by the Science and Engineering Research Council) in Hawaii, by a group including M.Burton, M.Bird,T.Geballe, A.Moorhouse, M.Toner, R.Wade, A.Webster, and P.Williams. Following the discovery of shocked molecular hydrogen in Orion (Gautier et al. 1976), difficulties faced by early hydrodynamic models (eg Hollenbach 1981) led to the general acceptance of magnetically-moderated C-shock models (Draine 1980, Draine \& Roberge 1982, Chernoff et al. 1982).

The results of our measurements are shown in figure 1. These inferred column densities cover a much wider range of level energy than previously used in model fits, and clearly are at variance with published C-shock models (the dashed lines). On the other hand, the cooling zone behind a hydrodynamic shock is modelled by the continuous line, and is evidently a good fit. Two parameters are required, the overall intensity (the curve is drawn through the strongest line 1-0 S(1) ) and the slope at high level energy which is determined by the pressure driving the shock.

That is the evidence that the position at which these data were obtained, Peak 1 in the Orion molecular outflow (Beckwith et al. 1978), is excited not by a C-shock but by a hydrodynamic (or J-) shock. The evidence that this situation is outflow-wide is 
Figure 1(top). Column density derived from the observations at Peak 1 in Orion divided by those expected from $2000 \mathrm{~K}$ gas. The dashed lines are C-shock models, the solid line is a Jshock model.

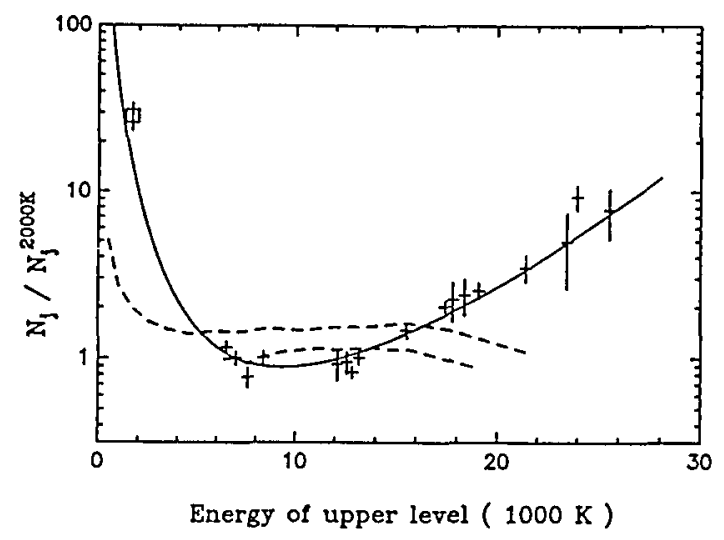

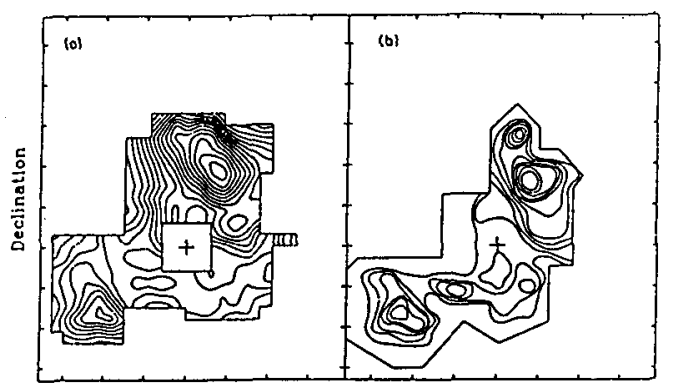

Right Ascension

Figure 2(centre left). $\quad 3.8 \mu \mathrm{m}$ map(left) and $2.1 \mu \mathrm{m}$ map from Beckwith et al(right) of the Orion molecular outflow.

Figure 3(centre right). The $3.8 \mu \mathrm{m}$ line ratio from all parts of the outflow, showing no trend with intensity.

Figure 4(bottom). The same as figure 1 , for data from the brightest part of the IC $443 \mathrm{H}_{2}$ emission
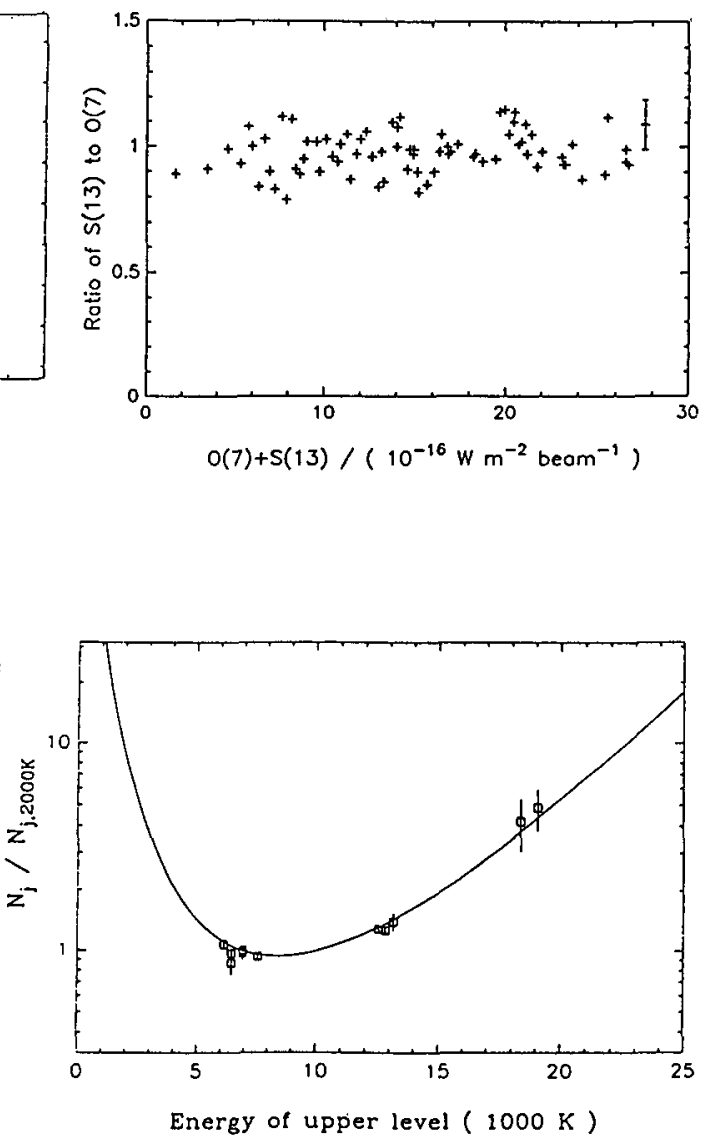
shown in figure 2, which consists of a map of intensity of the sum of two $3.8 \mu \mathrm{m}$ lines of $\mathrm{H}_{2}$ throughout the outflow. The right-hand map is drawn from the original $2.1 \mu \mathrm{m}$ map of Beckwith et al. for comparison. It is clear the morphology is the same. The lines in question are the 1-0 $O(7)$ line with an upper level energy of $8365 \mathrm{~K}$ and the 0-0 $\mathrm{S}(13)$ line with an upper level energy of $17445 \mathrm{~K}$. Because the wavelengths are nearly identical, there are no problems de-reddening the data. From figure 1 it is clear that the ratio is a sensitive indicator of the shape of the right-hand part of the model curve.

Figure 3 displays this ratio from all positions in this map, ordered according to intensity. It is clear that there is no significant trend in the data, and that the curve of figure 1 applies to all parts of the outflow. That is the evidence for J-shocks throughout the outflow.

Similar data obtained from the brightest part of the $\mathrm{H}_{2}$ emission in the supernova remnant IC 443 (Burton et al. 1988) is shown in figure 4. Again a J-shock-like pattern of column densities of $\mathrm{H}_{2}$ lines is produced. Burton et al. (1989) show that the line ratios in several other sources are consistent with this interpretation. These are the data on which is based the assertion that J-shock excitation appears to be common in shocked molecular clouds.

\section{THE THEORY}

Figure 5(a) is a diagram of the cross section through a hydrodynamic J-shock, showing the cooling zone where the temperature drops at a rate determined by the local cooling function. The cooling is supposed due to collisionally excited $\mathrm{H}_{2}$ either dissociating or de-exciting by line radiation, and to line emission from $\mathrm{CO}$ cooling. This can by crudely approximated by a steep power law, which is assumed in this argument. Figure 5(b) shows the same flow in temperature space, with superposed curves of the inverse cooling rate and a Boltzmann factor corresponding to a particular $\mathrm{H}_{2}$ line. The local density of excited molecules contains these as factors, and their product is also shown in figure $5(\mathrm{~b})$ as a dashed line. This peaks at a value depending only on the upper level energy of the line, and on the cooling function. It is clear from the diagram that for modest velocities (and therefore modest post-shock temperature maxima) that the integral under the dashed curve does not depend on velocity. In this approximation, and taking $s$ to be the $T$-exponent in the cooling function, the column densities of the various lines with upper level energies of $T_{j}$ are proportional to $\left(T_{j}\right)^{-8}$. This approximates closely to the solid curve in figure 1 . 

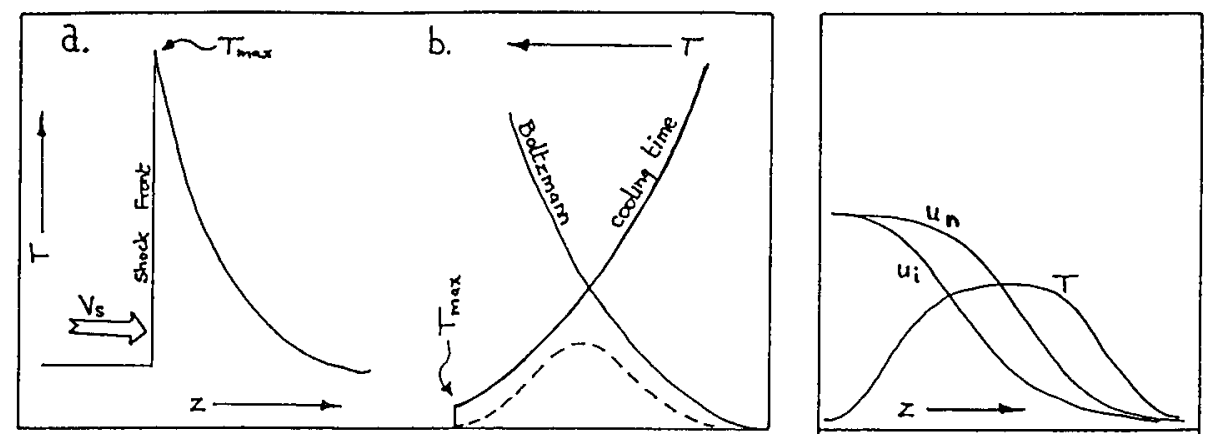

Figure 5(left). A schematic of a hydrodynamic (J-)shock. ' $a$ ' shows the temperature profile as a function of distance through the cooling zone, while ' $b$ ' is the same region in $T$-space showing the behaviour of $e^{-T_{j} / T}$ and of the cooling time of the shocked gas. Their product is shown as a dashed line.

Figure 6(right). shows the velocities of the ions $u_{i}$ and of the neutrals $u_{n}$, and of the temperature in the frame of a C-shock.

On the other hand, a C-shock structure is quite different. The heating is by friction with the streaming ions, and is relatively slow compared with the cooling time, in contrast to the $J$-shock case. This means that the region where the radiation is generated reaches its highest temperature when heating balances cooling, and it behaves like a slab of gas at that maximum temperature (figure 6). Since the heating is velocity dependent, so is the maximum temperature. Thus the expected behaviour of column densities in a C-shock is that it would represent a single temperature, dependent on the shock velocity. This seems to be at variance with our observations. Because C-shock models imply a lower density in the shocked gas (there is a longer distance over which a given line is radiated), these models also show signs that the highest $\mathrm{H}_{2}$ levels are not fully populated, and therefore the corresponding column densities fall below the Boltzmann line. On the other hand, the cooling time increases as temperature drops, and so the lowest levels find themselves in what is to all intents a cooling zone, with frictional heating no longer significant, and the pattern of column densities shows the characteristic rise at low excitation energies established also behind J-shocks. Both these phenomena are apparent in the C-shock model in figure 1. 


\section{INTERPRETATION}

If the evidence is accepted at face value, then there are several consequences. First of all, the very good fit of the model to the data in figure 1 implies that the cooling function is correct. This means that the cooling in these shocks is dominated by $\mathrm{H}_{2}$ dissociation above approximately $3000 \mathrm{~K}$, by $\mathrm{H}_{2}$ line cooling, and then by $\mathrm{CO}$ and other molecular dipole cooling below $1000 \mathrm{~K}$.

Secondly, the extinction to the Orion outflow is lower than generally accepted. The best fit value to these data is $A_{K}=0.8$, which implies a visual extinction of only 9 magnitudes between it and the Trapezium stars.

Thirdly, dissociational cooling depends on the square of the density while line cooling, being saturated, depends only on the first power of density. The slope of the cooling curve at high excitation energies is therefore steeper if the pressure (and therefore the density at a given temperature) behind the shock is greater. Correspondingly, the slope of the curve in figure 1 flattens. The best fit pressure at Peak 1 in Orion (or indeed throughout the Orion flow, since the $0-0 \mathrm{~S}(13)$ to $1-0 \mathrm{O}(7)$ ratio is constant) is high, with a value of $8 \times 10^{10} \mathrm{~K} \mathrm{~cm}^{-3}$. This provides fierce constraints on the properties of the wind driving the flow.

This seems a very neat package, but there are problems. The first is that measurements of rotationally excited $\mathrm{CO}$ in the Orion outflow made by several groups, and summarised by McKee (1982), indicate a post-shock density an order of magnitude lower than deduced from these models. However, the region from which this radiation was collected is much greater than the region delineated by the $\mathrm{H}_{2}$ peak, and within the vicinity there are several sources of $\mathrm{CO}$ heating, with very different properties. Further work is required.

The second problem appears to be a problem for any shock model, but is especially acute for $\mathrm{J}$-shock models, which completely dissociate $\mathrm{H}_{2}$ at shock speeds above $25 \mathrm{~km} \mathrm{~s}^{-1}$. The width of the profile observed (Brand et al. 1989) implies that in the line of sight there is a significant fraction of the excited gas travelling at up to \pm 100 $\mathrm{km} \mathrm{s}^{-1}$, i.e. at over three times the dissociation speed.

However, problems or no, it is clear that the capability of high performance spectrometers on a large IR telescope to obtain accurate intensities for $\mathrm{H}_{2}$ lines over a wide 
range of intensity and wavelength is providing an excellent basis for a new and detailed look at the mechanics of energy deposition in molecular clouds by young stellar objects.

\section{REFERENCES}

Beckwith, S., Persson, S.E., Neugebauer, G., \& Becklin, E.E. 1978, Ap. J. 234, L213.

Brand, P.W.J.L., Toner, M.P., Geballe,T.R., \& Webster, A.S., 1989, MNRAS 236, 929.

Burton, M.G., Geballe, T.R., Brand, P.W.J.L. \& Webster, A.S., 1988, MNRAS. 231, 617.

Burton, M.G., 1989, MNRAS. in press.

Chernoff, D.F., Hollenbach, D.J., \& McKee, C.F., 1982 Ap. J. 259, L97.

Draine, B.T., 1980, Ap. J., 2411021.

Draine, B.T., \& Roberge, W.G., 1982, Ap. J. 259, L91.

Gautier, T.N. III, Fink, U., Treffers, R.R., \& Larson H.P., 1976, Ap. J. 207, L29.

Hollenbach, D.J., 1981, Symposium on the Orion Nebula to honour Henry Draper, N.Y. Acad. Sci, New York, 242.

McKee, C.F., Storey, J.V.W., Watson, D.M., \& Green S., 1982, Ap. J. $259,647$.

\section{Discussion:}

MÜNCH: 1) How high is Boltzmann temperature $\mathrm{T}_{B}$ derived from the intensity ratio between the two lines in the $3.6 \mu \mathrm{m}$ range?. 2) How high is $\mathrm{T}_{B}$ and the J-number of the highest excitation rotational line?.

BRAND: 1) The temperature derived from the line ratio of the $\mathrm{H}_{2}$ lines $\emptyset-\emptyset \mathrm{S}(13)$ and $1-\emptyset \mathrm{O}(7)$, is $2800 \mathrm{~K} .2)$ The highest angular quantum number we have observed is $\mathrm{J}=19$, $\emptyset-\emptyset \mathrm{S}(17)$. The excitation temperature is $25541 \mathrm{~K}$. The equivalent observed Boltzmann temperature is aproximately $3500 \mathrm{~K}$. These temperatures are only loosely relates to the temperature of the emitting gas, which varies from a very high temperature just behind the shock to very low temperature after a few cooling lengths. Gas at all temperatures contributes to each line, in a manner determined by the cooling rate, and the excitation temperature of the line. 